Mhicrowave Engineering

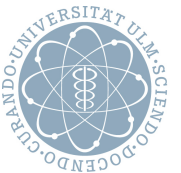

\title{
Expanding the Unambiguous Velocity Limitation of the Stepped-Carrier OFDM Radar Scheme
}

Benedikt Schweizer, Daniel Schindler, Christina Knill, Jürgen Hasch, and Christian Waldschmidt

(C) 2018 IEEE. Personal use of this material is permitted. Permission from IEEE must be obtained for all other uses, in any current or future media, including reprinting/republishing this material for advertising or promotional purposes, creating new collective works, for resale or redistribution to servers or lists, or reuse of any copyrighted component of this work in other works.

DOI: 10.23919/EuRAD.2018.8546621 


\title{
Expanding the Unambiguous Velocity Limitation of the Stepped-Carrier OFDM Radar Scheme
}

\author{
Benedikt Schweizer ${ }^{\# 1}$, Daniel Schindler ${ }^{* 2}$, Christina Knill ${ }^{\# 3}$, Jürgen Hasch ${ }^{* 4}$, Christian Waldschmidt ${ }^{\# 5}$ \\ \#Institute of Microwave Engineering, Ulm University, 89081 Ulm, Germany \\ *Corporate Sector Research and Advance Engineering of Robert Bosch GmbH, 70465 Stuttgart, Germany \\ $\left\{{ }^{1}\right.$ benedikt.schweizer, ${ }^{3}$ christina.knill, ${ }^{5}$ christian.waldschmidt $\} @$ uni-ulm.de, \\ $\left\{{ }^{2}\right.$ daniel.schindler, ${ }^{4}$ juergen.hasch $\} @$ de.bosch.com
}

\begin{abstract}
The main limitation for the practical implementation of OFDM radars is the required high sampling rate of $\mathrm{AD} / \mathrm{DA}$ converters. It can be solved by using a stepped-carrier OFDM scheme. Thereby, the same range and Doppler resolution is obtained as for a standard OFDM scheme at the cost of a reduced unambiguously measurable velocity. This paper presents a method to determine the actual velocity even for targets that violate the unambiguous velocity limitation. It is based on characteristics of the DFT and it is suitable for scenes with few targets or if all targets have a similar velocity.

Keywords - automotive, DFT, frequency-agility, OFDM, radar, stepped carrier
\end{abstract}

\section{INTRODUCTION}

Future radar applications require sensors that deliver a high resolution in range, Doppler, and angle at low cost. Orthogonal Frequency-Division Multiplexing (OFDM) is a promising approach to deal with those demands. It solves inherent hardware-specific limitations of classic frequency modulated schemes in the generation of highly accurate ramps and shifts the signal generation and evaluation to the digital domain. Furthermore, it allows to generate orthogonal waveforms such that a true multiple-input multiple-output (MIMO) radar, where all transmitters operate simultaneously, can be realized [1], [2]. The symbol duration is of few microseconds which results in a large unambiguously measurable range and velocity. The practical limitation of a conventional OFDM system [3] is the requirement of analog-to-digital (ADC) and digital-to-analog converters (DAC) with sampling rates of some gigahertz, depending on the desired range resolution. However, it is introduced in [4], [5] and extended in [6] that a stepped-OFDM scheme with an agile carrier that alters after each symbol, forming a regular pattern, can solve this constraint at the cost of reduced unambiguity in velocity. In this paper, a method is proposed that allows to determine the velocity of a target even if it is above the unambiguous velocity limitation of the stepped-carrier OFDM scheme. The approach, that was already applied to chirp-sequence based time-division multiplexing(TDM)-MIMO radars to improve angle estimation [7], is based on a phase error that occurs in the velocity calculation via DFT. The concept is validated with simulations and verified with measurements of a realistic traffic scenario.

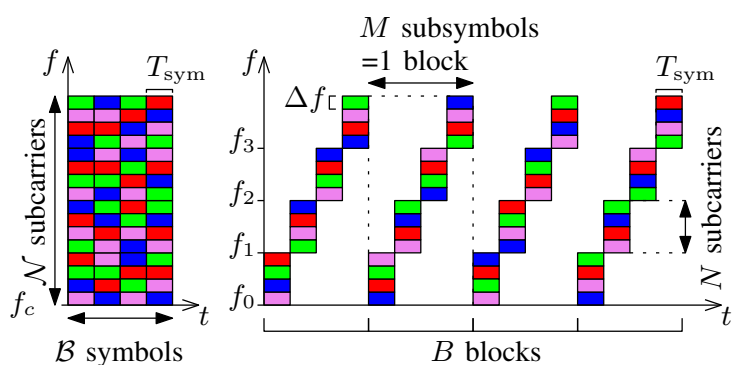

Fig. 1. Structure of OFDM signals in frequency domain, colors represent QPSK codes. Left: Standard OFDM. Right: Stepping-scheme in RF domain [6].

\section{STEPPED-CARRIER OFDM SCHEME}

OFDM waveforms can be realized using an IDFT, resembling the ideal signal

$$
x(t)=\sum_{n=0}^{N-1} d_{n} \mathrm{e}^{\mathrm{j} 2 \pi n \Delta f t} \text { rect }\left(\frac{t}{T_{\text {sym }}}\right) .
$$

of $N$ subcarriers that are spaced by $\Delta f$ in frequency and that are orthogonal due to the choice of the symbol duration $T_{\text {sym }}$ as $T_{\text {sym }}=1 / \Delta f$. Each subcarrier is coded with a QPSK-modulation symbol $d_{n}$. For practical use, a cyclic prefix of duration $T_{\mathrm{cp}}$ is added to avoid inter-symbol interference leading to the total duration $T=T_{\text {sym }}+T_{\text {cp }}$. For a standard OFDM scheme, $\mathcal{B}$ such symbols are transmitted consecutively but with different phase codes $d_{n b}$ to form the OFDM transmit signal.

To reduce the baseband bandwidth, the stepped-carrier OFDM uses $M$ symbols of smaller bandwidth, further referred to as subsymbols, that are transmitted at different carrier frequencies according to

$$
f_{m}=f_{c}+m N \Delta f
$$

to generate a larger measurement bandwidth in the channel, as explained in detail in [6]. $M$ such subsymbols form a block and $B$ blocks are transmitted consecutively, as illustrated in Fig. 1 for the standard and the stepped-carrier OFDM scheme.

At the receiver the signal is converted to baseband with the same carrier frequency $f_{m}$ such that the baseband bandwidth is relatively small. The received signal is a delayed and attenuated 


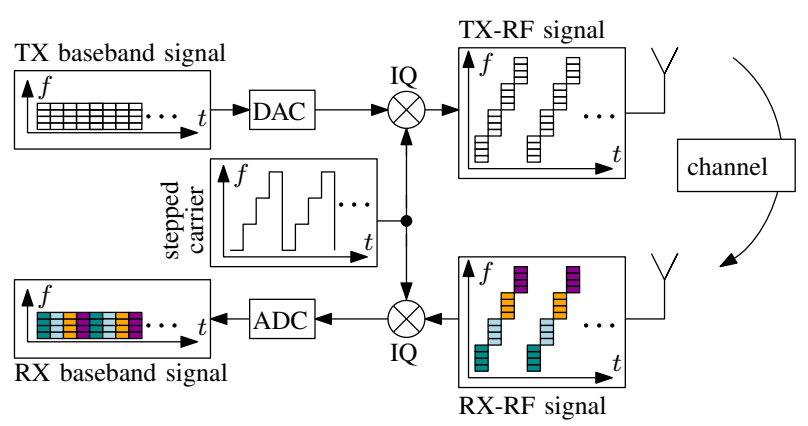

Fig. 2. Time-frequency pattern of the stepped-OFDM radar scheme to visualize the channel/baseband bandwidth and the stepped carrier. Colors represent the channel information included in the received modulation symbols.

version of the transmit signal and experienced a Doppler shift in case of a moving target. Due to the stepped-carrier, each subsymbol comprises the target information of the time instance and at the carrier frequency where it was actually transmitted. This is visualized in Fig. 2 by use of different colors. In frequency domain and after spectral division by the transmitted phase codes $d_{n m b}$, a matrix $D$ of modulation symbols

$$
\begin{aligned}
D_{n m b}= & \mathrm{e}^{-\mathrm{j} 2 \pi \frac{2 R_{0}}{c_{0}}\left(f_{c}+(n+m N) \Delta f\right)} \\
& \times \mathrm{e}^{\mathrm{j} 2 \pi \frac{2 v(m+b M) T}{c_{0}}\left(f_{c}+(n+m N) \Delta f\right)}
\end{aligned}
$$

depending on the range $R_{0}$, radial velocity $v$, and speed of light $c_{0}$ is present.

The range-Doppler evaluation of a conventional OFDM scheme is done by applying a DFT over all symbols to obtain $\mathcal{N}$ velocity profiles, one for each subcarrier, that is followed by a second IDFT in frequency direction for range evaluation.

As derived in [6], a modified DFT in the form

$$
V_{n m}[k]=\sum_{b=0}^{B-1} D_{n m b} \mathrm{e}^{-\mathrm{j} 2 \pi \frac{k(m+b M)}{M B}}, \quad k \in[0, B-1],
$$

is required to obtain velocity profiles that are aligned to the same reference point and consider the time instance when the symbols were actually transmitted. In that way, $N M$ velocity profiles are obtained on which the range evaluation can be performed.

The modified DFT can be reduced to a conventional DFT by rearranging the matrix of modulation symbols $D$ in a two-dimensional matrix $D_{2 \text { dim }}$ according to their actual time-frequency position in the channel. This corresponds to a variable substitution according to $\eta=n+m N$ and $\mu=m+b M$ what is equivalent to interlaced zeropadding. This can be seen as the generation of virtual subcarriers.

For a target with radial velocity $v$, the resulting velocity profiles of each virtual subcarrier have a maximum for the index

$$
k^{*}=\frac{2 v}{c_{0}} T\left(f_{m}+n \Delta f\right) M B
$$

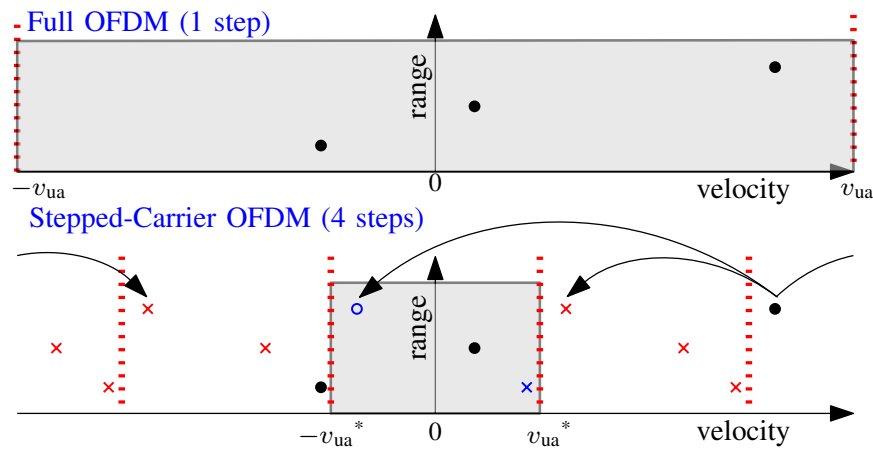

Fig. 3. Schematic range-velocity profile for standard (top) and stepped-carrier OFDM (bottom). The shadowed area represents the unambiguous velocity region. Targets $(\bullet)$ are folded back to the unambiguous region $(\times, 0)$ and by use of a conventional DFT, the profile is repeated $M$ times (indicated by dotted red lines and $\times$ ).

The range evaluation is done using all $N M$ velocity profiles by applying an IDFT in direction of $\eta$ for each velocity bin $k \in[0, B-1]$. Thus, the resulting range-velocity profile is of size $N M \times B$

\section{Ambiguous Velocity Estimation}

As only each $M^{\text {th }}$ modulation symbol is available on each subcarrier $\eta$ over time compared to a standard OFDM scheme, the Doppler frequency is sampled with a reduced sampling rate. This results in a reduction of the unambiguously measurable Doppler frequency by $M$ as can be retraced in Fig. 3.

If a conventional DFT of size $M B$ is used in combination with the rearranged matrix $D_{2 \mathrm{dim}}$, the theoretical maximum velocity of the DFT corresponds to the one of the standard OFDM scheme. Thus, the effective size of the profile is $B$ instead of $M B$ and they are spectrally repeated $M$ times in the profile obtained with the conventional DFT.

Consequently, the resulting peak in the range-velocity profile of a target with a velocity that is larger than the maximum unambiguous velocity is folded back to the unambiguous domain, as visualized in Fig. 3 for three targets that are in different unambiguous areas. Additionally, it shows the reduction of the unambiguous region and the spectral repetitions. As a consequence, a target with velocity $v=v_{0}$ and another one with e.g. $v=v_{0}+2 v_{\text {ua }}$ appear at the same position in the range-velocity profile.

Furthermore, the range-velocity profile is corrupted with an additional smear in range direction for an ambiguous velocity, as it can be seen in Fig. 4, where a simulated range-velocity profile of two targets with velocities $v_{1}=2 \mathrm{~m} / \mathrm{s}$ and $v_{2}=8.75 \mathrm{~m} / \mathrm{s}$ is shown. The maximum unambiguous velocity is $v_{\text {ua }}= \pm 7.07 \mathrm{~m} / \mathrm{s}$. Thus, the ambiguous velocity $v_{2}$ is visible at $v_{2}{ }^{*}=v_{2}-2 v_{\text {ua }}=-5.39 \mathrm{~m} / \mathrm{s}$.

The behavior can be explained with the result of the velocity calculation via a modified DFT for a target that has an ambiguous velocity of $v=v_{0}+\xi 2 v_{u a}$, where $\xi$ describes the 


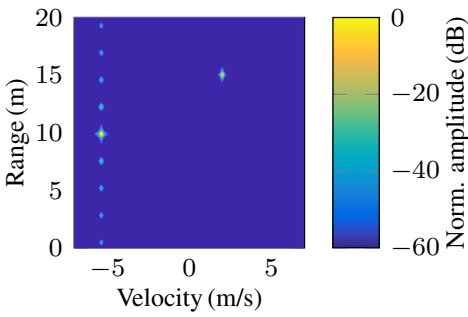

Fig. 4. Original situation: Simulated range-velocity profile for two targets with unambiguous velocity $v_{1}=2 \mathrm{~m} / \mathrm{s}$ at $r_{1}=15 \mathrm{~m}$ and ambiguous velocity $v_{2}=8.75 \mathrm{~m} / \mathrm{s}$ at $r_{2}=10 \mathrm{~m}$. The resulting peak of target 2 is folded into the unambiguous region $\left(v_{2}^{*}=-5.39 \mathrm{~m} / \mathrm{s}\right)$ and causes artifacts in range direction. Simulation parameters are listed in Table 1.

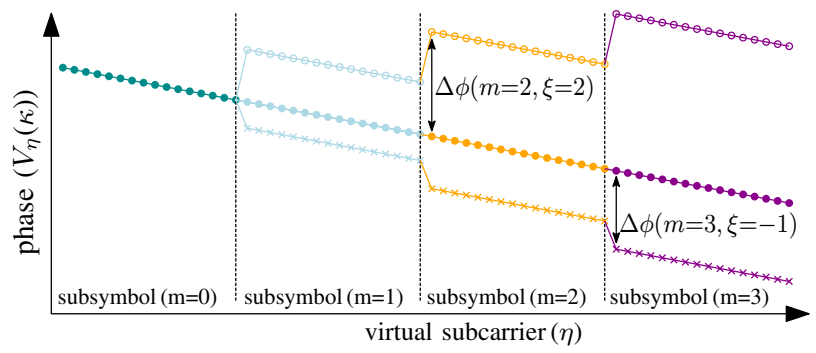

Fig. 5. Phase of virtual subcarriers $\eta$ including range information $(\bullet, \xi=0)$ and additional phase error $\Delta \phi$ for targets with $\xi=-1(x)$ and $\xi=2(0)$. The colors represent the involved subsymbols and thereby the time-frequency position, according to Fig. 2. The phase error is observable at the transition between subcarriers belonging to different subsymbols.

order of the spectral repetition. This corresponds to a shift of $\xi B$ for the index $k$ and the resulting Doppler profiles are

$$
V_{\eta}[k+\xi B]=\sum_{b=0}^{B-1} D_{n m b} \mathrm{e}^{-\mathrm{j} 2 \pi k(m+b M) T} \cdot \mathrm{e}^{\mathrm{j} 2 \pi \xi m / M},
$$

depending on $\eta=n+m N$, as there exists a velocity profile for each virtual subcarrier.

The peak appears at the expected index $k$ corresponding to velocity $v_{0}$, but the velocity profiles comprise an additional phase term

$$
\Delta \phi=\mathrm{e}^{\mathrm{j} 2 \pi \xi m / M}
$$

that depends on the spectral repetition $\xi$, the number of subsymbols $M$, and the set of subsymbols $m$.

This additional phase term is noticeable, if all $N M$ velocity profiles are used to evaluate the range information at index $k^{*}$ where a target is present. Then, a phase jump is observable that is superimposed on the range information at the transition from subcarrier $m N$ to $m N+1$, i.e., between velocity profiles that belong to different carrier positions. This behavior is depicted in Fig. 5 for 16 subcarriers and 4 subsymbols. The decreasing phase corresponds to a specific range for a target inside the unambiguous area, and the additional phase jump is exemplarily shown for one target with $v=v_{0}-2 v_{\text {ua }}(\xi=-1)$ and one target with $v=v_{0}+4 v_{\text {ua }}(\xi=2)$ in addition to the undisturbed case of a target inside the unambiguous region.

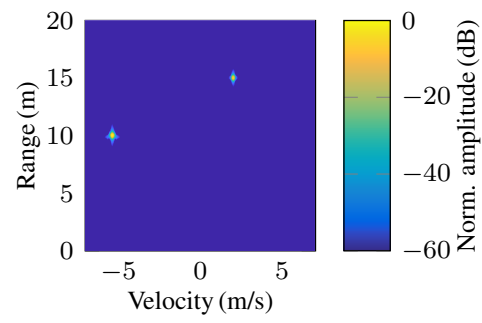

Fig. 6. Corrected situation: Simulated range-velocity profile for two targets. The velocity of the target at $r_{2}=10 \mathrm{~m}$ is larger than the unambiguous velocity, but the incorporated phase offset was detected and corrected and thereby the artifacts are removed.

\section{PROCESSING SCHEME}

The phase error $\Delta \phi$ is a characteristic that allows to determine the actual velocity of a target if it is larger than the unambiguous velocity.

Furthermore, with the information of the phase jump, it is not only possible to determine but also to correct the phase error such that the range-velocity profile shows a sharp peak without artifacts.

Therefore, $\xi$ is determined for each discrete velocity of the unambiguous velocity interval. If the correct repetition $\xi$ is known, the phase offset can be corrected before the range compression is performed and a range-velocity profile without artifacts is obtained. Combining the range-velocity profile with the phase information allows to assign the correct velocity to all targets.

The scenario from Fig. 4 is used to demonstrate the correction. By applying the phase offset detection on the velocity profiles, it can be determined that $\xi$ is -1 for the target at $r_{1}=10 \mathrm{~m}$ and the velocity profiles may be corrected such that a error-free range-velocity profile is obtained as shown in Fig. 6.

As the correction is done for each discrete velocity index of the velocity profiles and it is only possible to assign one spectral repetition $\xi$ to each velocity bin, the scheme can only deal with one actual velocity per velocity index, i.e., all targets folded back to that cell need to be of the same spectral repetition. However, there may be multiple targets at various distances in each velocity cell, as long as they all have a velocity of the same spectral repetition. Furthermore, the scheme is only capable of determining the ambiguous velocity of a target, but is not able to tell if there are multiple targets of different spectral repetitions at the same position in the $r-v$ diagram.

\section{Measurements}

To verify the processing scheme, a $77 \mathrm{GHz}$ OFDM radar demonstrator [6] based on measuring devices is used. It has a heterodyne structure with a digital IF stage, where the stepped-carrier is applied and uses two different RF carrier frequencies for up and down conversion such that the sidebands do not overlap and a velocity estimation is possible 


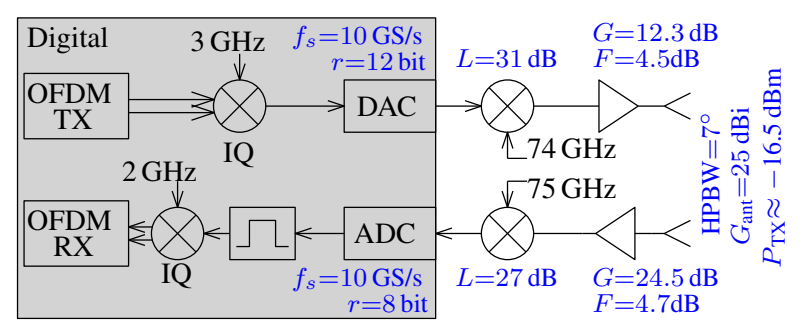

Fig. 7. Schematic of the heterodyne OFDM radar demonstrator at $77 \mathrm{GHz}$ [6].

Table 1. Parameters of stepped-carrier OFDM radar used for measurements and simulations. They are optimized to yield a low unambiguous velocity and do not represent a practical parameterization.

\begin{tabular}{|c|c|c|}
\hline Subsymbols / Steps & $M$ & 16 \\
\hline Subcarriers & $N$ & 512 \\
\hline Blocks & $B$ & 56 \\
\hline RF bandwidth $(\mathrm{GHz})$ & $\mathcal{W}$ & 1.024 \\
\hline Baseband bandwidth $(\mathrm{GHz})$ & $W$ & 0.064 \\
\hline Subcarrier spacing $(\mathrm{kHz})$ & $\Delta f$ & 125 \\
\hline Duration of cyclic prefix $(\mu \mathrm{s})$ & $T_{\mathrm{cp}}$ & 0.6 \\
\hline Unambiguous range $(\mathrm{m})$ & $R_{\mathrm{ua}}$ & 1200 \\
\hline Maximum range $(\mathrm{m})$ & $R_{\mathrm{max}}$ & 90 \\
\hline Range resolution $(\mathrm{m})$ & $\Delta R$ & 0.147 \\
\hline Unambiguous velocity $(\mathrm{m} / \mathrm{s})$ & $v_{\text {ua }}$ & \pm 7.07 \\
\hline Velocity resolution $(\mathrm{m} / \mathrm{s})$ & $\Delta v$ & 0.257 \\
\hline
\end{tabular}

without IQ mixers in the RF stage. The main parameters are specified in Fig. 7, where also the setup is shown in detail.

The parameters of the stepped-OFDM scheme, which were also used for the simulations, are chosen such that the unambiguous velocity $v_{\text {ua }}=7.07 \mathrm{~m} / \mathrm{s}$ is very small. This allows to show the correction in a traffic environment for an approaching car with $v=30 \mathrm{~km} / \mathrm{h}$. To make the artifacts more prominent, the signal-to-noise ratio (SNR) is increased by mounting an additional corner reflector to the front of the car.

As expected, the velocity is ambiguous. Hence, only a spectral repetition is observable in the range-velocity profile in Fig. 8. After determining the spectral repetition of the target to $\xi=-1$, the undisturbed profile as shown in Fig. 9 can be obtained. The actual velocity of the target may be calculated by combining the velocity of the range-velocity profile with the order of the spectral repetition to

$$
v_{\text {target }}=v_{\text {rv-profile }}-2 \xi v_{\text {ua }} \text {. }
$$

\section{CONCLUSION}

The stepped-carrier OFDM radar scheme is a realizable variant to a standard OFDM scheme of large baseband bandwidth at the cost of reduced unambiguous velocity. This limitation can partly be solved by exploiting characteristics of the DFT during velocity evaluation which allow to determine the actual velocities of targets that fold into the unambiguous

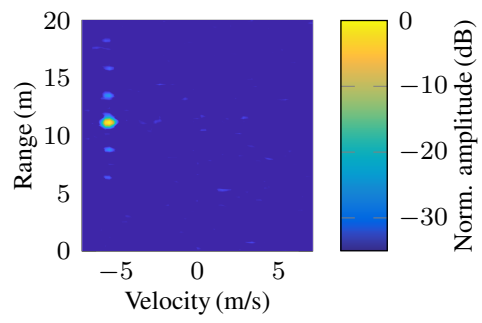

Fig. 8. Original measurement: Approaching car with $v=8.75 \mathrm{~m} / \mathrm{s}$. Due to the limited unambiguous velocity it appears as a spectral repetition at $v=-5.39 \mathrm{~m} / \mathrm{s}$ and causes artifacts.

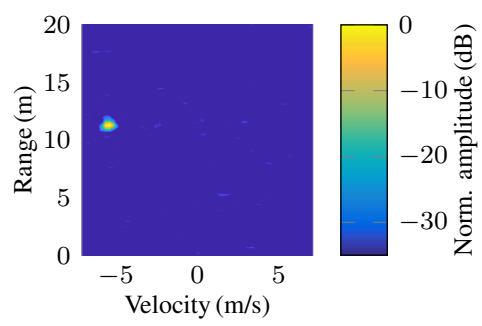

Fig. 9. Corrected measurement: The phase error is detected and it is determined that $\xi=-1$. After correction, the range-velocity profile is free of artifacts. The correct velocity of $v=8.75 \mathrm{~m} / \mathrm{s}$ is known by combining the profile with the information $\xi=-1$.

region. The method is applicable in scenarios with few targets or if all targets have a similar velocity.

\section{ACKNOWLEDGMENT}

The authors would like to thank the German Federal Ministry of Education and Research (BMBF), which partly funded the activities presented in this paper within the scope of the projects THINGS2DO (16ES0505) and autoSWIFT (16ES03).

\section{REFERENCES}

[1] B. Donnet and I. Longstaff, "Combining MIMO radar with OFDM communications," in Proc. 2006 Eur. Radar Conf., pp. 37-40.

[2] C. Sturm, Y. L. Sit, M. Braun, and T. Zwick, "Spectrally interleaved multi-carrier signals for radar network applications and multi-input multi-output radar," IET Radar, Sonar Navig., vol. 7, no. 3, pp. 261-269, Mar. 2013.

[3] C. Sturm, E. Pancera, T. Zwick, and W. Wiesbeck, "A novel approach to OFDM radar processing," in Proc. 2009 IEEE Radar Conf., pp. 1-4.

[4] G. Lellouch, A. K. Mishra, and M. Inggs, "Stepped OFDM radar technique to resolve range and Doppler simultaneously," IEEE Trans. Aerosp. Electron. Syst., vol. 51, no. 2, pp. 937-950, Apr. 2015.

[5] K. Huo, B. Deng, Y. Liu, W. Jiang, and J. Mao, "The principle of synthesizing HRRP based on a new OFDM phase-coded stepped-frequency radar signal," in Proc. 10th Int. Conf. Signal Process. IEEE, Oct. 2010, pp. 1994-1998.

[6] B. Schweizer, C. Knill, D. Schindler, and C. Waldschmidt, "Stepped-carrier OFDM-radar processing scheme to retrieve high-resolution range-velocity profile at low sampling rate," IEEE Trans. Microw. Theory Tech., vol. 66, no. 3, pp. 1610-1618, Mar. 2018.

[7] F. Roos, J. Bechter, N. Appenrodt, J. Dickmann, and C. Waldschmidt, "Enhancement of Doppler unambiguity for chirp-sequence modulated TDM-MIMO radars," in Proc. IEEE MTT-S Int. Conf. Microw. Intell. Mobility (ICMIM), 2018, pp. 25-28. 